\title{
Erratum to: Considerations of Human Health Risk Assessment in Chemical Accident: Suggestions from a Toxicogenomic Approach
}

\author{
Jun Hyuek Yang ${ }^{1}$, Hyun Soo Kim', Bon Kon Koo², \\ Cheol Min Lee ${ }^{3}$, Jong-Hyeon Jung ${ }^{4} \&$ \\ Young Rok Seo ${ }^{1}$
}

${ }^{1}$ Department of Life Science, Institute of Environmental Medicine for Green Chemistry, Dongguk University Biomedi Campus, Goyang-si, Gyeonggi-do 10326, Republic of Korea

${ }^{2}$ Vision inside, Inc., 24, Kyungheedae-ro 1na-gil, Dongdaemun-gu Seoul 02454, Republic of Korea

${ }^{3}$ Department of Chemical and Biological Engineering, Seokyeong University, Seoul, Republic of Korea

${ }^{4}$ Faculty of Health Science, Daegu Haany University, Gyeongsan 38610, Republic of Korea

Correspondence and requests for materials should be addressed to Y. R. Seo (seoyr@dongguk.edu)

Received 6 November 2017 / Received in revised form 17 April 2018

Accepted 14 May 2018

DOI 10.1007/s13530-018-0350-8

(C)The Korean Society of Environmental Risk Assessment and

Health Science and Springer 2018

pISSN : 2005-9752 / elSSN : 2233-7784

Toxicol. Environ. Health. Sci. Vol. 10(2), 79-89, 2018

In the 2018 issue of Toxicology and Environmental Health Sciences (ToxEHS), an error occurred in the research article. Jun Hyuek Yang, Hyun Soo Kim, Bon Kon Koo, Cheol Min Lee, Jong-Hyeon Jung \& Young Rok Seo (2018) Considerations of Human Health Risk Assessment in Chemical Accident: Suggestions from a Toxicogenomic Approach Toxicol. Environ. Health Sci. 10(2), 79-89
- In the section of acknowledgment

Original:

This study was supported in part by grant (17-105-001) from the Korea Ministry of Environment.

This should be replaced by:

This study was supported by grant (2017001970002) from the Korea Ministry of Environment through "The Chemical Accident Prevention Technology Development Project". 